\title{
Acantholytic squamous cell carcinoma: Pathological study of three cases in Ecuador
}

\author{
Eduardo Garzón Aldas*, Gabriela Torres
}

Clínica Dermatológica Garzón, Ecuador

\begin{abstract}
Adenoid squamous cell carcinoma is an uncommon histopathological variant of squamous cell carcinoma characterized by acantholysis of tumor cells creating pseudolumina and the appearance of glandular differentiation. It is also known as pseudoglandular, pseudoangiosarcomatous, adenoid, epithelioma dyskeratoticum, or adenoacanthoma. In this article, we describe and analyze three cases of acantholytic cell carcinoma. The purpose is to verify if the data of our cases can be validated with scientific information. All our cases presented lesions in the face, and in one of them the developed tumor on the skin showed actinic keratosis. The other two tumors were located in the right side of the face, and both were big: the bigger tumor was approximately $8 \times 4 \mathrm{~cm}$ and the other one with a size of $6 \times 2.5 \mathrm{~cm}$. Due to the size and location, they were both send to the oncologist for treatment. One of our cases showed metatypical carcinomas accompanied by acantholytic variant of squamous cell carcinoma. None of our cases presented metastasis. The histopathological finding were keratinized squamous tumor cell type, adenoid structures with round spaces with a defined wall of at least one-cell width, and spaces with isolated or group of dyskeratotic cells.
\end{abstract}

Keywords: acantholytic squamous cell carcinoma; adenoid; metatypical carcinoma

Citation: Aldas EG and Torres G. Acantholytic squamous cell carcinoma: Pathological study of three cases in Ecuador. J Surg Dermatol 2021; 6(1): 151; http://dx.doi.org/10.18282/jsd.v6.i1.151.

*Correspondence to: Eduardo Garzón Aldas, Clínica Dermatológica Garzón, Calle Farina e Ilaló Esquina, Edificio Mariana de Jesús, Tercer piso, Valle de los Chillos, Ecuador; eduderma@hotmail.com

Received: $6^{\text {th }}$ November 2020; Accepted: $18^{\text {th }}$ December 2020; Published Online: $10^{\text {th }}$ January 2021

\section{Introduction}

The acantholytic squamous cell carcinoma was initially described by Lever in 1947, and represents a malignant epithelial with gland-like and solid pattern, with extension into the dermis known also as adenoacanthoma of the sweat glands. Subsequently, it was decided to change his scaly concept with the cellular acantholytic phenomenon that gave it an adenoid aspect ${ }^{[1,2]}$.

This variant develops predominantly in areas of sun exposure on the body ${ }^{[3]}$, although it has also been described in mamas ${ }^{[4]}$, vulva ${ }^{[5]}$, penis ${ }^{[6]}$, conjunctiva ${ }^{[7]}$, nasopharynx ${ }^{[8]}$ and mouth ${ }^{[9]}$.

In 1967, Müller found microscopic features such as pseudoglandular elements, dyskeratosis, acantholysis, atypical mitosis, and perineural extension, and it has been considered as a rare variant of squamous cell carcinoma (SCC). It has received other names such as adenocarcinoma of sweat glands ${ }^{[10]}$; however, it is well established that it is not a sweat gland carcinoma ${ }^{[11]}$. The term dyskeratotic endocrine cell carcinoma (ECC) is also inadequate since many squamous cell carcinomas present with dyskeratotic cells ${ }^{[10]}$. The term acantholytic ECC emphasizes the observed acantholytic phenomenon. The term pseudoglandular ECC emphasizes that the tumor, although it is epidermal, is capable to form structures that adopt an adenoid pattern such as occurs with adenoid basal cell carcinoma or adenoid seborrheic keratosis ${ }^{[12]}$.

Histologically, the tumor is composed of strands and islands of atypical epithelial cells extending into the dermis. Connection to the overlying epidermis is seen in most cases, which may show hyperkeratosis and parakeratosis ${ }^{[13]}$. However, this connection may be only focal or, in some cases, absent ${ }^{[1]}$. Many of the tumor 
strands may show tubular and alveolar formations, which are referred to as pseudoglandular appendages. These spaces contain acantholytic cells that result from loss of cohesion of the tumor cells ${ }^{[3]}$. These acantholytic cells may appear extremely bizarre, large, or multinucleated. Mitotic figures are variably present. Classic SCC may also show cleft formation with dyskeratosis and acantholysis, but it does not have a definite wall or cohesive layer of cells surrounding the acantholytic cells ${ }^{[11]}$.

Squamous cell carcinoma is the second most frequent skin cancer in Ecuador. An Ecuadorian study done in 2009 found the following percentages: basocellular carcinoma $58 \%$, squamous cell carcinoma $34 \%$, and melanoma $8 \%$. Squamous cell carcinoma shows an incidence of $1 \%$ in South America. Its variant acantholytic cell carcinoma is a very rare entity, and at the moment, there is no data in Ecuador of the incidence of this cancer ${ }^{[14]}$.

Due to its rarity and lack of epidemiological information, we chose to describe our three cases of acantholytic squamous cell carcinoma and become the first authors to report this in Ecuador.

\section{Materials and methods}

We collected the patients' data in our private practice (age, gender, race, occupation, location of tumor, and macroscopic description (Table 1)) for acantholytic squamous cell carcinoma. Informed consent was taken in all three patients for the making of the present study. The reports mentioned are from patients in the age range between 56 and 75 years, with 2:1 gender ratio (women/ men), and all of them with lesion in the face. After the biopsy, the samples were fixed with $10 \%$ buffered formalin and sent for histopathological processing using paraffin inclusion and hematoxylin-eosin staining. Also, immunohistochemical tests for carcinoembryonic antigen (CEA), S100, and amylase were requested for all three patients.

\section{First case}

Our first case was a 65-year-old man, farmer, Caucasian, phototype IV, with a history of hypertension and controlled gastritis. He came in December 2015 with a giant tumor on his right cheek of eight months of evolution. Macroscopically the tumor was red colored, fixed, with hard consistence, of $8 \mathrm{~cm} \times 4 \mathrm{~cm}$ size, and with no cervical adenopaties found (Figure 1).

Microscopally we found hyperkeratosis in the papillary and reticular dermal thickness, and epithelial cells in adenoid pattern, which were characteristics of acantholytic squamous cell carcinoma (Figure 2 and Figure 3).

Therefore, the tumor was diagnosed as an acantholytic squamous cell carcinoma. Immunohistochemical used for sweat gland carcinomas such as CEA, S-100, and amylase were negative. Due to the size of the tumor, he did not received treatment from us but was immediately transferred to the oncological service for treatment.

\section{Second case}

Our second patient was a 75 -year-old indigenous woman with photoype IV who works as a farmer and with a history of recurrent squamous cell carcinoma of the right cheek. At the moment, she presented a recurrent tumor, and it developed as an actinic keratosis. She had shown these recurrent lesions for one year. The lesion presented an ulceration area at the bottom. Macroscopally the tumor was $6 \times 2.5 \mathrm{~cm}$ in size, reddish and with soft consistence (Figure 4). This patient as well as the other one, showed no cervical or head adenopathies.

Microscopically we found keratinized squamous tumor cell type, adenoid structures with round spaces with a defined wall of at least one-cell width, and spaces with isolated or group of dyskeratotic cells (Figure 5 and Figure 6). Immunohistochemical used for sweat gland carcinomas such as CEA, S-100, and amylase were negative. This patient was also transferred to the oncology service for treatment.

Table 1. General characteristic of patients with acantholytic squamous cell carcinoma

\begin{tabular}{llll}
\hline Characteristic & Patient 1 & Patient 2 & Patient 3 \\
\hline Age & 65 & 75 & 57 \\
Gender & Male & Female & Female \\
Race & Caucasian & Indigenous & Caucasian \\
Occupation & Farmer & Farmer & Agriculture \\
Location & Face (right cheek) & Face (right cheek) & Face (nose) \\
Time of evolution & 8 months & 1 year & 3 months \\
Metastasis & Negative & Negative & Negative \\
Treatment & Transferred to oncology for & Transferred to oncology for & Patient lost \\
treatment & treatment & Acantholysis, pseudotubular \\
Histopathological finding & Acantholysis, pseudotubular & Dyskeratosis, acantholysis, & spaces, atypical keratinocytes \\
& spaces, atypical keratinocytes & keratinocytes & Negative \\
Immunohistology for CEA, S100, atypical \\
amylase
\end{tabular}




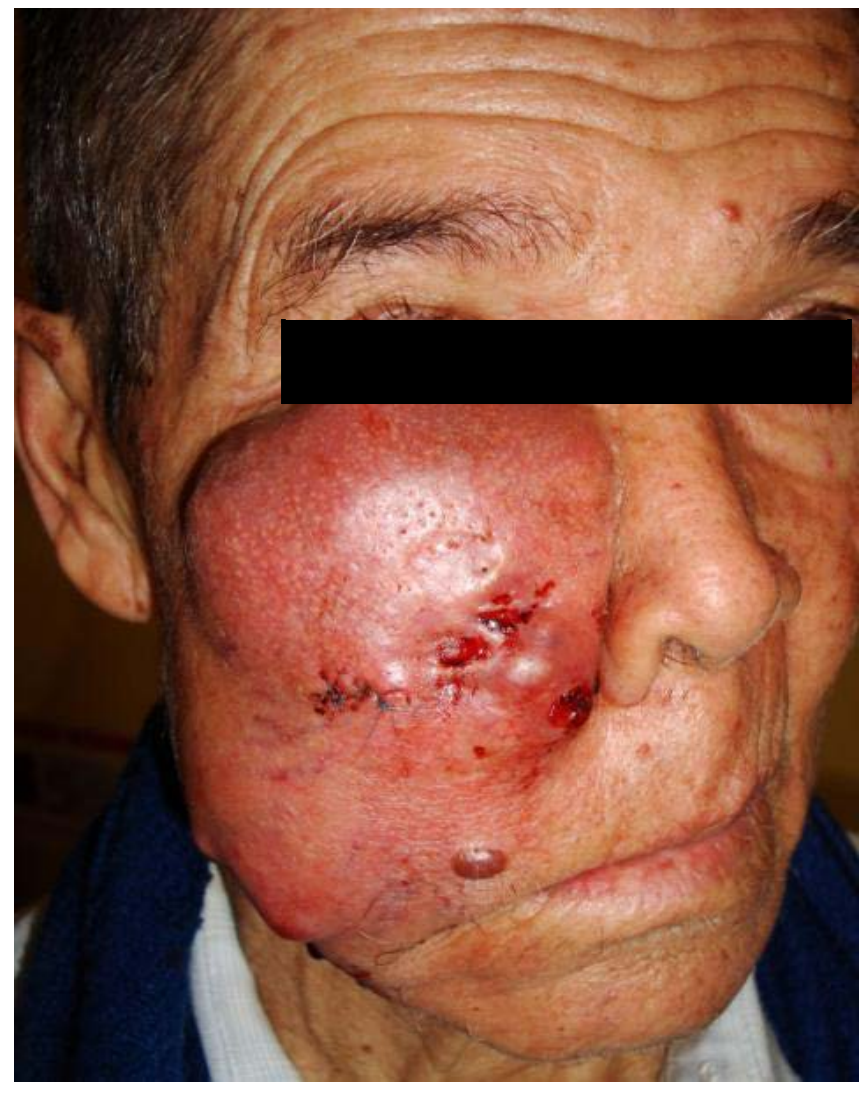

Figure 1. Exophytic giant tumor with hard consistence on the right cheek, the central site of biopsy

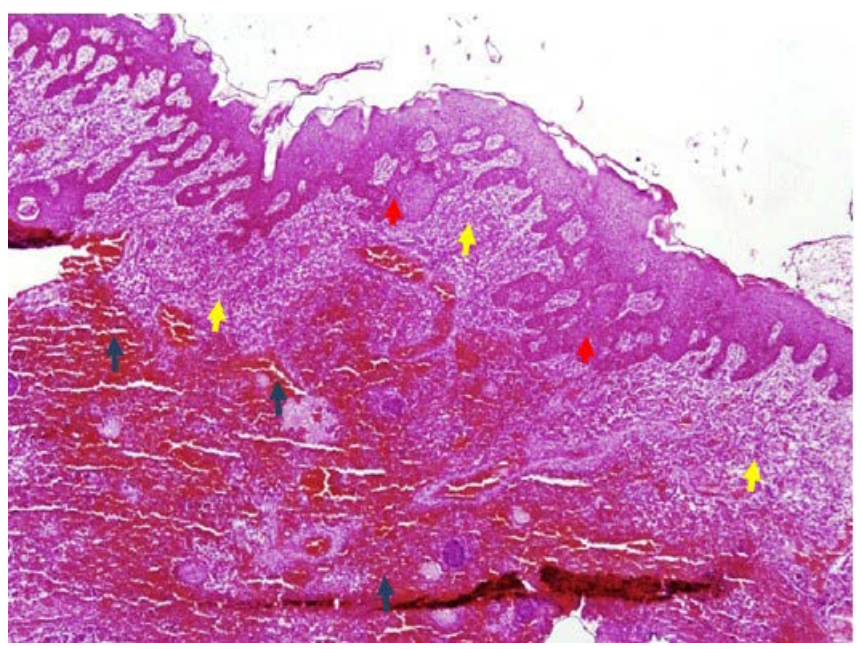

Figure 2. Evident pseudoepitheliomatous epidermal hyperplasia (red arrows), dermis with hemorrhagic areas (blue arrows) and occupied by neoplasic cellularity (yellow arrows) (hematoxylin and eosin, $5 \mathrm{X}$ )

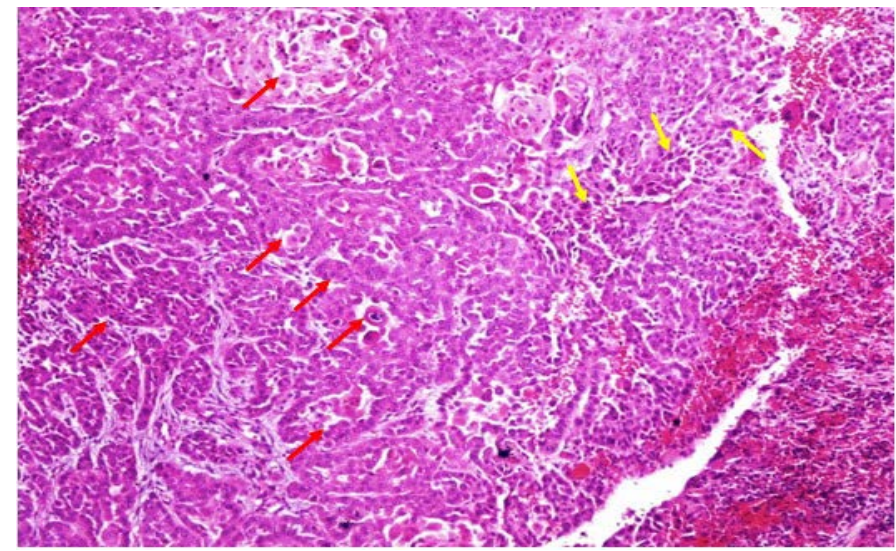

Figure 3. Dermal aggregates of squamous cells with hyperchromatic nuclei of different size (yellow arrows), among which are spaces that resemble adenoid structures (red arrows) (hematoxylin and eosin, 20X)

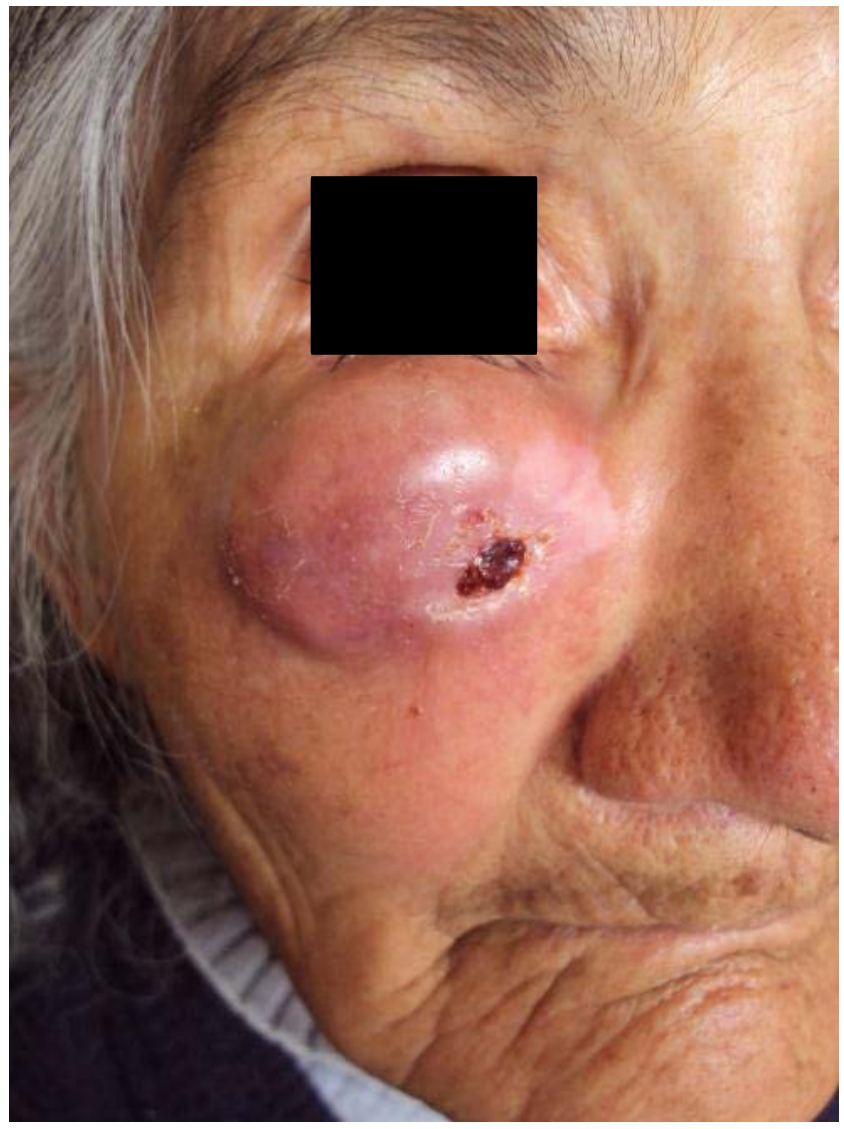

Figure 4. Exophytic giant tumor with hard consistence on the right cheek, the central site of biopsy 


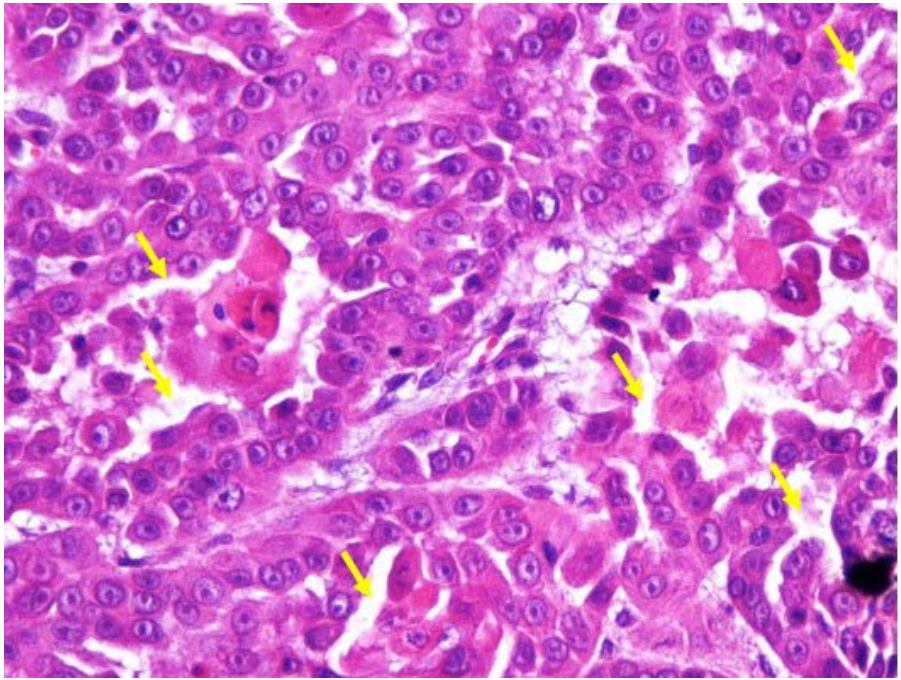

Figure 5. Detail of neoplastic keratinocytes with acantholytic spaces (arrows) (hematoxylin and eosin, 40X)

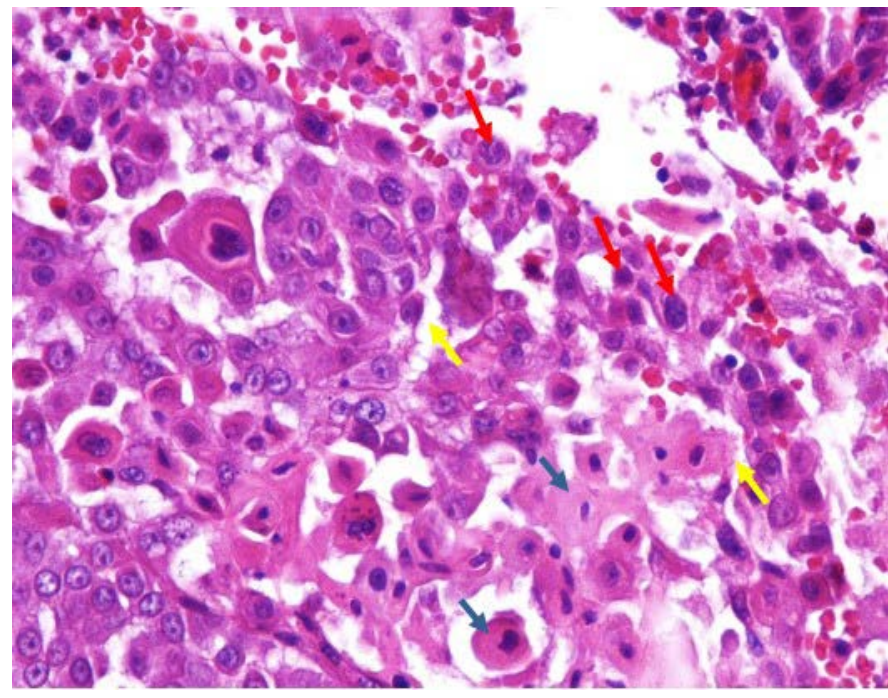

Figure 6. Dyskeratosis (blue arrows), acantholysis (yellow arrows), hyperchromasia, pleomorphism (red arrows) (hematoxylin and eosin, 100X)

\section{Third case}

Our third case was a Caucasian woman of 56 years of age, with phototype IV and no pathological history of importance, who works in agriculture with a sun exposure of approximately six hours daily. The patient had a nasal lesion (Figure 7A) of approximately three months of evolution. It was previously treated by a private physician with topical antibiotics without improvement, which was why it was referred to our dermatological center. The lesion was characterized by a $2-\mathrm{cm}$ plaque, hyperpigmented and with the presence of telangiectasias in its periphery (Figure 7B). In addition, the central ulcerated region of $3 \times 5 \mathrm{~mm}$ was friable and bleeding with ease. This was the only case that presented metatypical carcinomas plus acantholytic variant of squamous cell carcinoma.

Microscopally we found neoplastic aggregates of squamous cells with evident and multiple acantholytic spaces between them (Figure 8-Figure 10). Immunohistochemical tests for CEA, S-100, and amylase were negative. The patient never came back after the results. We tried to reach her but it seemed she moved out of town.

\section{Discussion}

The acantholytic-pseudoglandular variant of squamous cell carcinoma is an uncommon pathology, representing $2 \%-4 \%$, developing mainly in elderly persons, as it can be confirmed in his description of three cases. The tumor is said to be of good prognosis, and there is no greater tendency to metastasis compared to the usual $\mathrm{ECC}^{[5]}$. Tumors from other locations such as oral mucosa, vulva, and conjunctiva may behave aggressively ${ }^{[3]}$.

Clinically it appears as an ulcerated papula with a slow-growing pattern. The lesions typically arise on the head and neck as a nodule or ulcer on sun-exposed skin, particularly on and around the ears and face, and they may develop from an actinic keratosis as our third case.

The differential diagnosis includes basal cell carcinoma, eccrine adenocarcinoma, metastatic adenocarcinoma, and angiosarcoma, all of which have a pseudo-glandular or adenoid growth pattern. Immunostaining used for sweat gland carcinomas such as CEA, S-100, and amylase are not detectable in the pseudoglandular acantholytic $\mathrm{ECC}^{[4]}$. At high-power field, we can identify the malignant criteria of cells: voluminous, hyperchromatic, and pleomorphic nuclei presenting atypical mitosis ${ }^{[14]}$.

As regards the age of the patients, the youngest patient was 56-year-old and the oldest 75-year-old. Evolution period was known from three months to eight months. The male predominance could not be demonstrated since two of our patients were women.

Morphologically, all our cases presented significant acantholysis, pseudotubular structures with adenoidlike pattern, keratinocytic pleomorphism, and hyperchromasia. One of the tumors developed from an actinic keratosis, and metatypical signs with basocellular features were also seen in one of our patients.

The conventional treatment still remains the same as for the squamous cellular carcinoma, which consists of surgical excision of the tumor ${ }^{[13]}$. It is worth saying that in all of our cases, no metastases were found. The oncology department suggested radiotherapy for reducing the size of the tumors in patients 1 and 2, before surgery.

\section{Conclusions}

Acantholytic squamous cell carcinoma is a rare histopathological variety of spinocellular carcinoma which frequently develops in sun-exposed regions, 

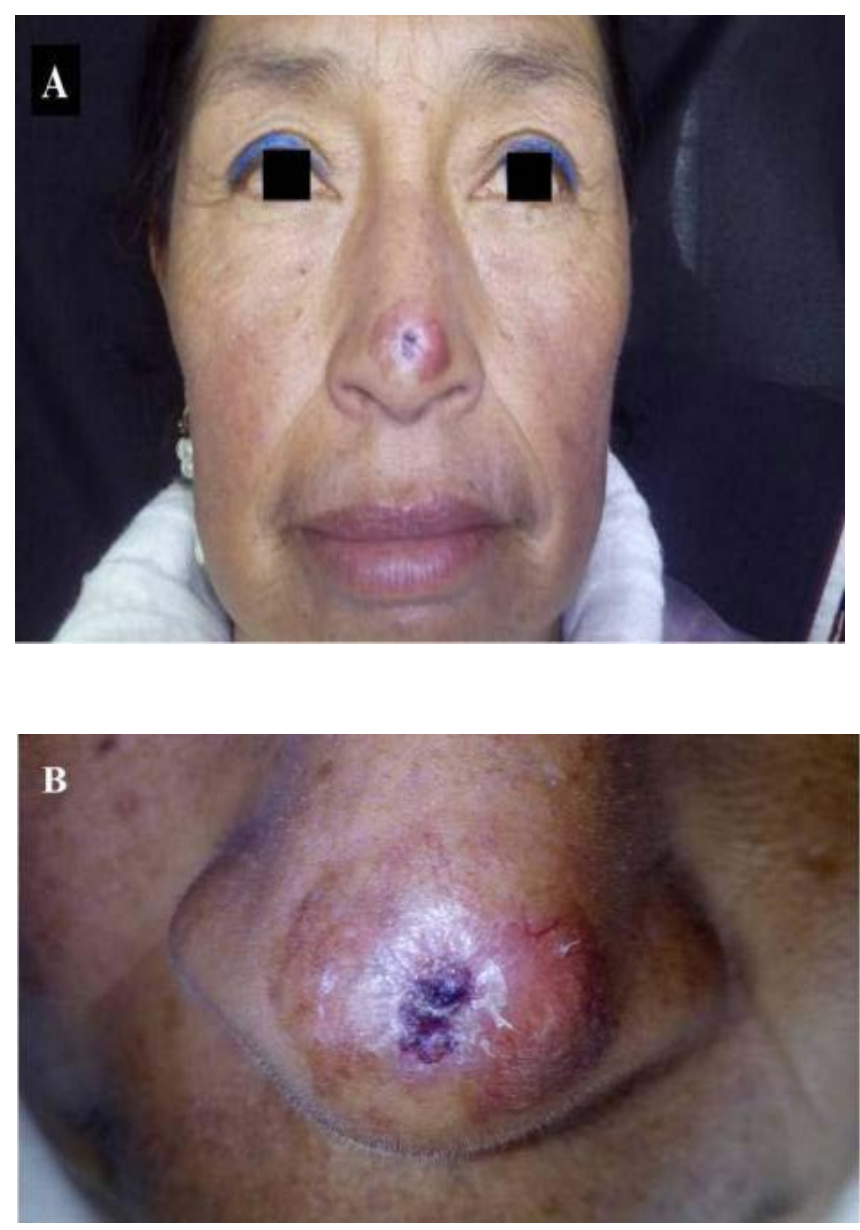

Figure 7. (A and B) Round nasal plaque with pearly edges and peripheral telangiectasias

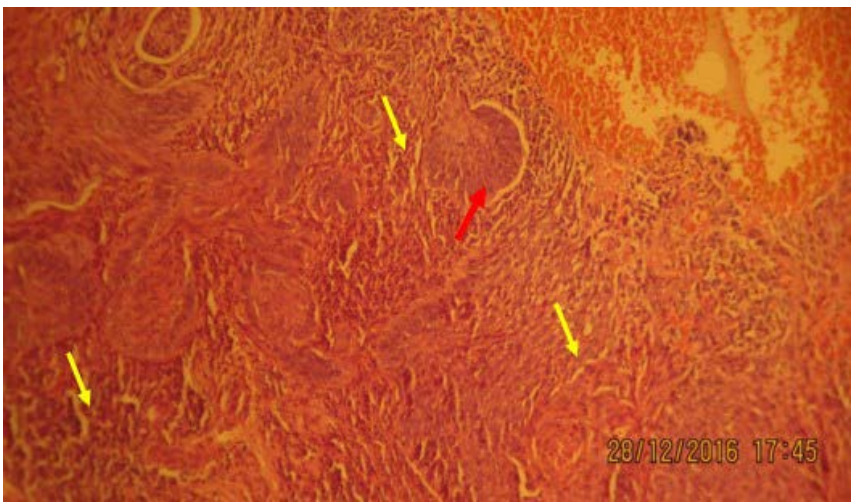

Figure 8. Neoplastic aggregates of squamous cells with evident and multiple acantholytic spaces between them (yellow arrows). We can also see a neoplastic aggregation of basal cells surrounded by stromal retraction (red arrows) (hematoxylin and eosin, 10X)

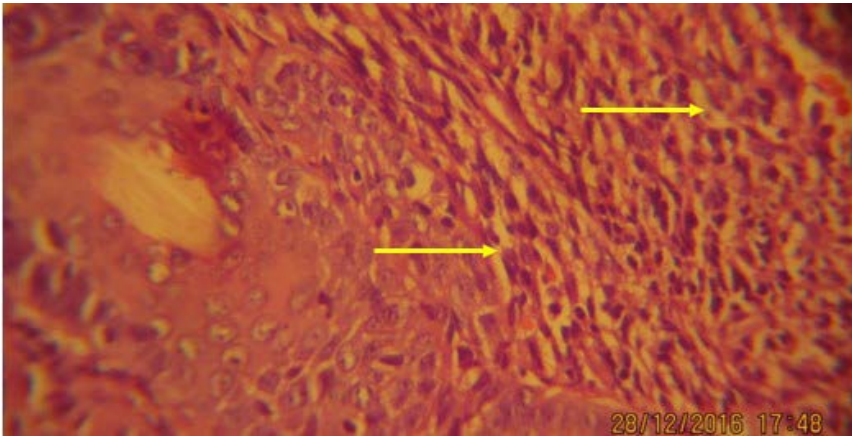

Figure 9. Acantholytic spaces within scaly aggregates (yellow arrows) (hematoxylin and eosin, 20X)

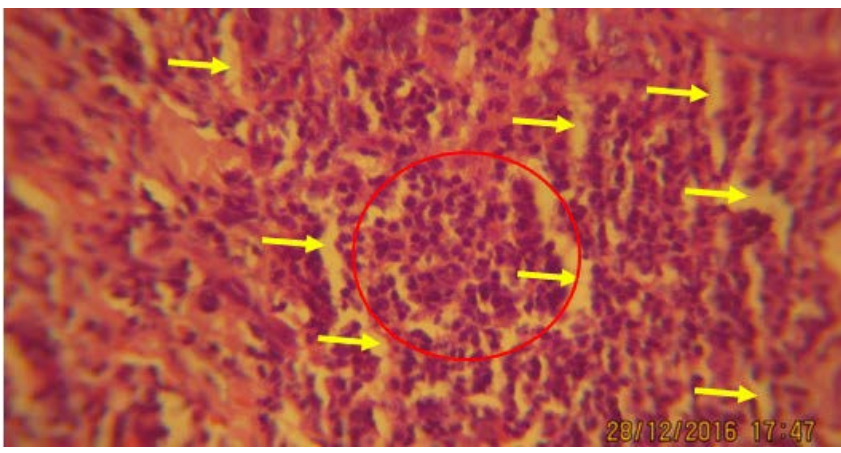

Figure 10. Hyperchromasia, pleomorphisms (circle), and absolute inter-queratinocytic acantholytic disgregation, resembling tubular structures or vascular spaces (yellow arrows) (hematoxylin and eosin, 20X)

especially the face, usually in the elderlies with preexisting lesions such as actinic keratosis ${ }^{[11]}$. There is controversy about its aggressivity; some say that the tumor has a well-known aggressivity and many others consider that it has a low aggressivity. It is important to consider differential diagnoses such as sebaceous carcinoma among others $^{[13]}$.

We suggest using the term pseudoglandularacantholytic squamous cell carcinoma, emphasizing its capacity to form glandular structures and the acantholytic phenomenon common to these tumors.

\section{Authors' contributions}

EG Aldas supervised two cases and performed the histopathological study of all cases reported here. DG Torres supervised one case and typed the article.

\section{Acknowledgments}

We would like to thank all the medical team of Dermatologic Garzón, and the doctors who registered the 
cases, took the pictures and searched for all the scientific information.

\section{Conflict of interest}

The authors declare no potential conflict of interest with respect to the research, authorship, and/or publication of this article.

\section{References}

1. Lever WF. Adenoacanthoma of sweat glands. Arch Derm Syphilol 1947; 56(2): 157-171. doi: 10.1001/archderm.1947.0 1520080017002.

2. Driemel O, Müller-Richter UD, Hakim SG, Bauer R., Berndt A, et al. Oral acantholytic squamous cell carcinoma shares clinical and histological features with angiosarcoma. Head Face Med 2008; 4: 17. doi: 10.1186/1746-160X-4-17.

3. Yu R, Gohla G, Alowami S. Pseudoglandular squamous cell carcinoma. Cutis 2015; 95(2): 68, 104-106.

4. Eusebi V, Lamovec J, Cattani MG, Fedeli F, Millis RR. Acantholytic variant of squamous-cell carcinoma of the breast. Am J Surg Pathol 1986; 10(2): 855-861. doi: 10.1097/00000478-198612000-00004.

5. Lasser A, Cornog JL, Morris JM. Adenoid squamous cell carcinoma of the vulva. Cancer 1974; 33(1): 224-227. doi: 10.1002/1097-0142(197401)33:1<224::AID-CNCR28203301 $33>3.0 . \mathrm{CO} ; 2-6$.

6. Watanabe K, Mukawa A, Miyazaki K, Tsukahara K. Adenoid squamous cell carcinoma of the penis. Report of a surgical case clinically manifested with rapid lung metastasis. Acta Pathol Jpn 1983; 33(6): 1243-1250. doi: 10.1111/j.1440-1827. 1983.tb02169.x.

7. Mauriello JA Jr, Abdelsalam A, McLean IW. Adenoid squamous carcinoma of the conjunctiva-a clinicopathological study of 14 cases. Br J Ophthalmol 1997; 81(11): 1001-1005. doi: $10.1136 /$ bjo.81.11.1001
8. Zaatari GS, Santoianni RA. Adenoid squamous cell carcinoma of the nasopharynx and neck region. Arch Pathol Lab Med 1986; 110(6): 542-546.

9. Papadopoulou E, Tosios KI, Nikitakis N, Papadogeorgakis N, Sklavounou-Andrikopoulou A. Acantholytic squamous cell carcinoma of the gingiva: Report of a case and review of the literature. Oral Surg Oral Med Oral Pathol Oral Radiol Endod 2010; 109(6): e67-e71. doi: 10.1016/j.tripleo.2010.01.019.

10. Lever WF. Adenoacanthoma of sweat glands. Archives of Dermatol and Syphil 1974; 57: 157-171.

11. Nappi O, Wick MR, Pettinato G, Ghiselli RW, Swanson PE. Pseudovascular adenoid squamous cell carcinoma of the skin. A neoplasm that may be mistaken for angiosarcoma. Am J Surg Pathol 1992; 16(5): 429-438. doi: 10.1097/00000478-19 9205000-00001.

12. Ball E, de Kok E, Tirado M, Lista MT. (Spanish) [Acantholytic pseudoglandular squamous cell cancer. Clinico-pathologic study of 36 cases and bibliographic review]. Dermatol Venez 2012; 50(1): 449-457.

13. Sajin M, Hodorogea Prisăcaru A, Luchian MC, Pătraşcu OM, Dumitru A, et al. Acantholytic squamous cell carcinoma: Pathological study of nine cases with review of literature. Rom J Morphol Embryol 2014; 55(2): 279-283.

14. Motley R, Kersey P, Lawrence C; British Association of Dermatologists; British Association of Plastic Surgeons, et al. Multiprofessional guidelines for the management of the patient with primary cutaneous squamous cell carcinoma. Br J Dermatol 2012; 146: 18-25. doi: 10.1046/j.0007-0963.2001.04 615.x. 